\title{
Gene polymorphisms of autophagy machinery and the risk of hepatitis $B$ virus-related hepatocellular carcinoma in a Thai population
}

\author{
Suthida Wisetsathorn ${ }^{\mathrm{a}}$, Varangkana Tantithavorn ${ }^{\mathrm{a}}$, Nattiya Hirankarn ${ }^{\mathrm{b}}$, Pisit Tangkijvanich ${ }^{\mathrm{c}}$, \\ Thammakorn Saethang ${ }^{\mathrm{d}}$, Ingorn Kimkong ${ }^{\mathrm{a}, \mathrm{e}, *}$ \\ ${ }^{a}$ Department of Microbiology, Faculty of Science, Kasetsart University, Bangkok 10900 Thailand \\ b Center of Excellence in Immunology and Immune Mediated Diseases, Department of Microbiology, \\ Faculty of Medicine, Chulalongkorn University, Bangkok 10330 Thailand \\ c Research Unit of Hepatitis and Liver Cancer, Department of Biochemistry, Faculty of Medicine, \\ Chulalongkorn University, Bangkok 10330 Thailand \\ d Systems Biology Center, Research Affairs, Faculty of Medicine, Chulalongkorn University, \\ Bangkok 10330 Thailand \\ e Center for Advanced Studies in Tropical Natural Resources, \\ National Research University-Kasetsart University, Kasetsart University, Bangkok 10900 Thailand
}

*Corresponding author, e-mail: fsciiok@ku.ac.th

Received 16 Jul 2017

Accepted 28 Nov 2017

\begin{abstract}
Most cases of hepatocellular carcinoma (HCC) are associated with hepatitis B virus (HBV) infection. Previous studies revealed that some autophagy-related genes expression was increased in the HCC cell lines and tissue samples when compared to the controls. However, the mechanism of these autophagy genes in HBV-related HCC is still unclear. Hence we aim to search for the functional single nucleotide polymorphisms (SNPs) of autophagy-related genes associated with HCC. Two hundred and sixteen Thai patients with chronic HBV (CHB) infection (102 with HCC and 114 without HCC), 91 recovered individuals, and 131 healthy controls were recruited. The PCR-RFLP method and TaqMan allelic discrimination assay were applied to analyse eight SNPs including mTOR rs2295080, ATG7 rs2305686, ATG5 rs41292420, ATG5 rs77859116, ATG5 rs510432, IGF1 rs75960260, PIK3C3 rs3813065, and ATG16L1 rs2241880. Our study found that the A allele of ATG16L1 rs2241880 was associated with an increased risk in HCC as compared to CHB patients without HCC and healthy controls $(\mathrm{OR}=1.62, P=0.033$; OR $=1.98, P=0.002$, respectively). The T allele of ATG5 rs77859116 was significantly associated with the risk to HCC as compared with CHB patients without HCC (OR $=23.61, P=0.000007)$. Furthermore, we found that the combination of CT (rs41292420), TT (rs77859116), and CC (rs510432) genotypes of ATG5 conferred the risk to HCC, with the OR of the high-risk to low-risk group being 16.42 and $P=0.003$. This study suggests that the polymorphisms of the ATG5 and ATG16L1 genes might be involved in HBV-related hepatocellular carcinogenesis in Thai. However, a functional study of these polymorphisms should be further confirmed.
\end{abstract}

KEYWORDS: ATG16L1, ATG5

\section{INTRODUCTION}

Although an implementation of the vaccination programs in many countries has resulted in a decrease in the incidence of acute hepatitis $\mathrm{B}$, there are still approximately 240 million hepatitis B virus (HBV) carriers worldwide. More than 650000 people die each year due to liver cirrhosis and hepatocellular carcinoma (HCC $)^{1}$. The pathogenic mechanisms responsible for these complications are uncertain.
However, a study in Chinese twins showed that the difference of host genetics was involved with chronic HBV infection ${ }^{2}$. Hence the polymorphisms of the host genetic factors might be affecting to the HBV-related hepatic complications.

Autophagy is a cellular process involving the degradation of a pathogen or dysfunctional cellular components. The initiation of the autophagy process is regulated by the induction of the IGF1 (insulin-like growth factor-1) and mTOR (mam- 
malian target of rapamycin) pathways ${ }^{3}$. Beside the inhibition of these regulators, several factors including nutrient depletion, hypoxia, drug, and radiation can also induce autophagy. The induction of autophagy results in the activation of the Beclin-1/ class III phosphatidylinositol 3-kinase (PIK3C3) protein complex, which is important for the autophagosome initiation. In the step of autophagosome formation, two ubiquitin-like conjugation systems are crucial. The first involves the covalently binding of autophagy-related genes (ATG) 12 to ATG5 and then interacts with ATG16 to form the ATG12ATG5-ATG16 complex. The second system is the ATG8/LC3 conjugated to plasma membrane phosphatidylethanolamine. In addition, several enzymes including ATG3, ATG4, and ATG7 are required for this autophagosome maturation process ${ }^{4,5}$.

Our previous studies have shown the involvement of autophagy in HBV infection and the development of HCC. Increased mRNA levels of ATG16L1 and mTOR genes in the HBV-transfected HepG2.2.15 cell line compared to the human liver epithelial cell line (THLE-2 cells) and human hepatoma cell line (HepG2 cells) were found. Furthermore, the ATG16L1 mRNA expression in the HepG2 cells was higher than in the THLE- 2 cells ${ }^{6}$. Recently, we also found that mRNA and the protein expressions of ATG5 and ATG12 were significantly increased in HepG2.2.15 cells and tumour liver tissues from HBV-related HCC patients when compared to the controls ${ }^{7}$. However, the ATG7 gene expression was down-regulated in HepG2.2.15 cells when compared to HepG2 cells ${ }^{8}$. Thus we hypothesize that the gene polymorphisms of this autophagy machinery might influence the susceptibility to chronic HBV infection and the development of HCC in the Thai population.

In this study, we selected eight functional single nucleotide polymorphisms (SNPs) of the mTOR, ATG7, ATG5, IGF1, PIK3C3, and ATG16L1 genes to investigate the effects on chronic HBV infection in Thai patients.

\section{MATERIALS AND METHODS}

\section{Subjects}

Two hundred and sixteen patients with chronic HBV infection from Chulalongkorn Memorial Hospital were recruited. These patients were divided into two groups including patients without HCC $(N=$ 114; 42 women and 72 men: mean age $\pm \mathrm{SD}=$ $49 \pm 12$ years $)$ and patients with HCC $(N=102 ; 23$ women and 79 men: mean age $\pm \mathrm{SD}=56.8 \pm 9.6$ years). The diagnosis of chronic hepatitis B was confirmed by seropositivity for the hepatitis B surface antigen ( $\mathrm{HBsAg}$ ) over a 6-month period and did not have any other types of liver diseases; such as, chronic hepatitis $\mathrm{C}$ or alcoholic liver diseases. In addition, all patients had increased serum alanine aminotransferase (ALT) and aspartate aminotransferase (AST) levels. The HCC patients were diagnosed by histopathology and/or a combination of a mass lesion in the liver from hepatic imaging and the serum alpha foetoprotein level was higher than $400 \mathrm{ng} / \mathrm{ml}$. The control groups were also divided into two groups. The first group was 131 ethnically and geographically matched (83 women and 48 men: mean age $\pm S D=23 \pm 12$ years) from healthy blood donors of the Thai Red Cross Society were recruited as the healthy control group and the second group was self-limited HBV patients (51 women and 40 men: mean age $\pm S D=48 \pm 14$ years) who were tested HBsAg negative and both HBV core antibody (anti-HBc) and HBV surface antibody (anti-HBs) positive, with normal liver function tests, and no history of HBV vaccination. The Ethics Committee of the Faculty of Medicine, Chulalongkorn University, Bangkok, Thailand approved the study and the subjects gave their informed consent.

\section{DNA extraction and genotyping study}

The DNA was extracted from the buffy coat collected with ethylenediaminetetraacetic acid (EDTA) as an anticoagulant, using the salting-out method ${ }^{9}$. The DNA was stored at $-20^{\circ} \mathrm{C}$ until used. Four SNPs composed of ATG16L1 rs2241880 (A/G), IGF1 rs75960260 (A/G), PI3KC3 rs3813065 (T/C), and ATG5 rs77859116 (C/T) were analysed by the polymerase chain reaction-restriction fragment length polymorphism method. The primer sequences and restriction enzymes were newly designed in this study (Table 1). The PCR conditions were predenaturation at $94^{\circ} \mathrm{C}$ for $5 \mathrm{~min}$, followed by $35 \mathrm{cy}$ cles of denaturation at $95^{\circ} \mathrm{C}$ for $30 \mathrm{~s}$, annealing at $67^{\circ} \mathrm{C}$ for $30 \mathrm{~s}$ and elongation at $72^{\circ} \mathrm{C}$ for $30 \mathrm{~s}$, with a final elongation at $72{ }^{\circ} \mathrm{C}$ for $5 \mathrm{~min}$. The PCR products were checked by $1 \%$ of agarose gel and visualized under UV light with ethidium bromide staining. The PCR products were digested by restriction endonucleases. The restriction fragments were separated by electrophoresis on $3 \%$ of agarose gel and visualized under UV light with ethidium bromide staining. Ten percent of the samples were confirmed by direct sequencing of the PCR products to verify the accuracy of genotyping.

The other four SNPs: mTOR rs2295080 (T/G), 
Table 1 Primer sequences and restriction enzymes used in this study.

\begin{tabular}{llll}
\hline Gene names & Primer names & Primer sequences $\left(5^{\prime} \rightarrow 3^{\prime}\right)$ & Restriction enzymes \\
\hline ATG16L1 & rs2241880-F & GAGCAGTAAACCTCTGCAATCC & SfaNI \\
& rs2241880-R & CAGAGCCAAAAGGTGGAAAG & \\
IGF1 & rs75960260-F & GGACACCCAGGCAGGTATGC & Tsp45I \\
& rs75960260-R & GTGAAAGACCACGAGCTCC & \\
PI3KC3 & rs3813065-F & TAGTCCTTTCCCTACTC & SwaI \\
& rs3813065-R & CCAGTTTTCCTGTCTTG & HpyCH4IV \\
\hline
\end{tabular}

ATG5 rs510432 (T/C), ATG5 rs41292420 (T/C), and ATG7 rs2305686 (T/C), were genotyped by the Taqman SNP genotyping assay (Applied Biosystems). The PCR reactions were performed in 96well plates on the StepOnePlus Real-Time PCR System (Applied Biosystems). Each well contained 10 ng genomic DNA, TaqMan Genotyping Master Mix (Applied Biosystems), TaqMan genotyping assay Specific Probe mix (Applied Biosystems) and RNase Free water. One non-template-control well was included on each plate. After the DNA amplification $\left(95^{\circ} \mathrm{C}\right.$ for $10 \mathrm{~min}$, followed by 40 cycles of $95^{\circ} \mathrm{C}$ for $15 \mathrm{~s}$ and $60^{\circ} \mathrm{C}$ for $1 \mathrm{~min}$ ), fluorescence was acquired and analysed for allelic discrimination using the STEPONE software.

\section{Statistical analysis}

The genotype frequencies were checked for consistency among the normal controls with those expected from the Hardy-Weinberg equilibrium (HWE). The allele and genotype frequencies were compared between groups using the chi-squared $\left(\chi^{2}\right)$ test or Fisher's exact probability test, where appropriate. The PLINK v1.9 and EPI INFO v.7.2 programs were used to calculate HWE, $p$-values, odds ratios (ORs), and haplotype analysis ${ }^{10}$. The $p$-value $<0.05$ was considered significant. In addition, gene interaction was analysed by the multifactor dimensionality reduction (MDR) approach ${ }^{11}$.

\section{RESULTS}

In this study, we examined the effect of eight polymorphisms of the genes involved in autophagy regulation (mTOR and IGF1) and autophagy-related genes (PIK3C3, ATG5, ATG7, and ATG16L1) on chronic HBV infection and the development of HCC. The distribution of the allele and genotype frequencies was shown in Table 2 and Table 3, respectively. The control genotype frequencies of all SNPs were
HWE $(P>0.05)$. The results showed that the T allele of ATG5 rs77859116 was significantly associated with the risk to HCC as compared with CHB patients without HCC (OR $=23.61, P=0.000007)$. The effect of the $\mathrm{T}$ allele was similar to an autosomal recessive disorder, in which the presence of the TT genotype, when compared to TC and CC, conferred an OR of 25.00, $P=0.0000048$. Furthermore, the A allele of ATG16L1 SNP rs2241880 was associated with an increased risk in HCC when compared to CHB patients without HCC and healthy controls $(\mathrm{OR}=1.62, P=0.033 ; \mathrm{OR}=1.98, P=0.002$, respectively). The effect of the A allele seemed to be autosomal recessive of AA when compared with GG plus AG genotypes (CHB with HCC versus $\mathrm{CHB}$ without HCC and healthy control; $\mathrm{OR}=1.95, P=0.016$ and $\mathrm{OR}=2.43, P=0.0009$, respectively). However, we did not find any significant associations of the other 6 SNPs in the mTOR (rs2295080), ATG7 (rs2305686), ATG5 (rs41292420 and rs510432), IGF1 (rs75960260), and PIK3C3 (rs3813065) genes between patients with chronic HBV and the control groups.

Furthermore, we performed a haplotype analysis of these eight SNPs by comparing each tested haplotype with other haplotypes between the patients and the controls. In this study, no significant association between the haplotypes of the 8 SNPs and risk to disease development was found (Table 4). However, we applied the MDR method to investigate the interaction of the ATG5 gene polymorphisms including rs41292420, rs77859116, and rs510432. In this analysis, it was found that the combination of CT (rs41292420), TT (rs77859116), and CC (rs510432) genotypes of the ATG5 gene was classified as a high-risk group to HCC (ratio of case/control > 1) (Table 5). From the whole statistical analysis, the OR for the high-risk to lowrisk group was 16.42 and $P=0.003$. 
Table 2 Allele frequencies of eight polymorphisms of autophagy genes in chronic HBV patients (with HCC and without HCC), self-limited HBV patients and healthy controls.

\begin{tabular}{lcccccc}
\hline SNP & Allele & Total CHB & CHB w HCC & CHB w/o HCC & Self-limited HBV & Healthy controls \\
& & $N=216(\%)$ & $N=102(\%)$ & $N=114(\%)$ & $N=91(\%)$ & $N=131(\%)$ \\
\hline MTOR rs2295080,G/T & $\mathrm{G}$ & $111(25.7)$ & $57(27.9)$ & $54(23.7)$ & $50(27.5)$ & $64(24.4)$ \\
& $\mathrm{T}$ & $321(74.3)$ & $147(72.1)$ & $174(76.3)$ & $132(72.5)$ & $198(75.6)$ \\
ATG7 rs2305686, T/C & $\mathrm{T}$ & $124(28.7)$ & $52(25.5)$ & $72(31.6)$ & $60(33.2)$ & $79(30.2)$ \\
& $\mathrm{C}$ & $308(71.3)$ & $152(74.5)$ & $156(68.4)$ & $122(66.9)$ & $183(69.9)$ \\
ATG5 rs41292420, T/C & $\mathrm{T}$ & $34(7.9)$ & $17(8.3)$ & $17(7.5)$ & $10(5.5)$ & $16(6.1)$ \\
& $\mathrm{C}$ & $398(92.1)$ & $187(91.7)$ & $211(92.5)$ & $172(94.5)$ & $246(93.9)$ \\
ATG5 rs77859116, C/T & $\mathrm{C}$ & $12(2.8)$ & $0(0.0)$ & $12(5.3)$ & $0(0.0)$ & $0(0.0)$ \\
& $\mathrm{T}$ & $420(97.2)$ & $204(100.0)$ & $216(94.7)$ & $182(100.0)$ & $262(100.0)$ \\
ATG5 rs510432, T/C & $\mathrm{T}$ & $192(44.4)$ & $90(44.1)$ & $102(44.4)$ & $77(42.3)$ & $132(50.4)$ \\
& $\mathrm{C}$ & $240(55.6)$ & $114(55.9)$ & $126(55.3)$ & $105(57.7)$ & $130(49.6)$ \\
IGF1 rs75960260, A/G & $\mathrm{A}$ & $9(2.1)$ & $5(2.5)$ & $4(1.8)$ & $6(3.3)$ & $4(1.5)$ \\
& $\mathrm{G}$ & $423(97.9)$ & $199(97.6)$ & $224(98.3)$ & $176(96.7)$ & $258(98.5)$ \\
PIK3C3 rs3813065, T/C & $\mathrm{T}$ & $103(23.8)$ & $44(21.6)$ & $59(25.9)$ & $54(29.7)$ & $58(22.1)$ \\
& $\mathrm{C}$ & $329(76.2)$ & $160(78.4)$ & $169(74.1)$ & $128(70.3)$ & $204(77.9)$ \\
ATG16L1 rs2241880, G/A & $\mathrm{G}$ & $107(24.8)$ & $41(20.1)$ & $66(29.0)$ & $38(20.9)$ & $87(33.2)$ \\
& $\mathrm{A}^{\mathrm{b}}$ & $325(75.2)$ & $163(79.9)$ & $162(71.1)$ & $144(79.1)$ & $175(66.8)$ \\
\hline
\end{tabular}

$\mathrm{SNP}=$ single nucleotide polymorphism; $\mathrm{CHB}=$ chronic hepatitis $\mathrm{B}$; HBV = hepatitis B virus; $\mathrm{HCC}=$ hepatocellular carcinoma.

a Compared with C allele (CHB with HCC versus CHB without HCC); OR=23.61, $P=0.000007$.

${ }^{\mathrm{b}}$ Compared with G allele (CHB with HCC versus $\mathrm{CHB}$ without HCC and healthy control); OR = 1.62, $P=0.033$ and $\mathrm{OR}=1.98, P=0.002$, respectively.

Table 3 Genotype frequencies of eight polymorphisms of autophagy genes in chronic HBV patients (with HCC and without HCC), self-limited HBV patients and healthy controls.

\begin{tabular}{lcccccc}
\hline SNP & Genotype & $\begin{array}{c}\text { Total CHB } \\
N=216(\%)\end{array}$ & $\begin{array}{c}\text { CHB w HCC } \\
N=102(\%)\end{array}$ & $\begin{array}{c}\text { CHB w/o HCC } \\
N=114(\%)\end{array}$ & $\begin{array}{c}\text { Self-limited HBV } \\
N=91(\%)\end{array}$ & $\begin{array}{c}\text { Healthy controls } \\
N=131(\%)\end{array}$ \\
\hline MTOR rs2295080,G/T & GG & $13(6.0)$ & $7(6.9)$ & $6(5.37)$ & $8(8.8)$ & $7(5.3)$ \\
& GT & $85(39.4)$ & $43(42.2)$ & $42(36.8)$ & $34(37.4)$ & $50(38.2)$ \\
& TT & $118(54.6)$ & $52(51.0)$ & $66(57.9)$ & $49(53.9)$ & $74(56.5)$ \\
ATG7 rs2305686, T/C & TT & $16(7.4)$ & $6(5.9)$ & $10(8.8)$ & $11(12.1)$ & $14(10.7)$ \\
& TC & $92(42.6)$ & $40(39.2)$ & $52(45.6)$ & $38(41.8)$ & $51(38.9)$ \\
& CC & $108(50.0)$ & $56(54.9)$ & $52(45.6)$ & $42(46.2)$ & $66(50.4)$ \\
ATG5 rs41292420, T/C & TT & $1(0.5)$ & $0(0.0)$ & $1(0.9)$ & $2(2.2)$ & $1(0.8)$ \\
& TC & $32(14.8)$ & $17(16.7)$ & $15(13.2)$ & $6(6.6)$ & $14(10.8)$ \\
& CC & $183(84.7)$ & $85(83.3)$ & $98(86.0)$ & $83(91.2)$ & $116(88.6)$ \\
ATG5 rs77859116, C/T & CC & $0(0.0)$ & $0(0.0)$ & $0(0.0)$ & $0(0.0)$ & $0(0.0)$ \\
& CT & $12(5.6)$ & $0(0.0)$ & $12(10.5)$ & $0(0.0)$ & $0(0.0)$ \\
& TT & $204(94.4)$ & $102(100.0)$ & $102(89.5)$ & $91(100.0)$ & $131(100.0)$ \\
ATG5 rs510432, T/C & TT & $40(18.5)$ & $21(20.6)$ & $19(16.0)$ & $21(23.1)$ & $31(23.7)$ \\
& TC & $112(51.85)$ & $48(47.1)$ & $64(56.1)$ & $35(38.5)$ & $70(53.4)$ \\
IGF1 rs75960260, A/G & CC & $64(29.6)$ & $33(32.4)$ & $31(27.9)$ & $35(38.5)$ & $30(22.9)$ \\
& AA & $2(0.9)$ & $1(1.0)$ & $1(0.9)$ & $0(0.0)$ & $0(0.0)$ \\
PIK3C3 rs3813065, T/C & AG & $5(2.3)$ & $3(2.9)$ & $2(1.8)$ & $6(6.6)$ & $4(3.1)$ \\
& GG & $209(96.8)$ & $98(96.1)$ & $111(97.4)$ & $85(93.4)$ & $127(97.0)$ \\
& TT & $15(6.9)$ & $6(5.9)$ & $9(7.9)$ & $8(8.8)$ & $7(5.3)$ \\
ATG16L1 rs2241880, G/A & $73(33.8)$ & $32(31.4)$ & $41(36.0)$ & $38(41.8)$ & $44(33.6)$ \\
& CC & $128(59.3)$ & $64(62.8)$ & $64(56.2)$ & $45(49.5)$ & $80(61.1)$ \\
& GG & $10(4.6)$ & $4(3.9)$ & $6(5.3)$ & $3(3.3)$ & $11(8.4)$ \\
& GA & $87(40.3)$ & $33(32.4)$ & $54(47.4)$ & $32(35.2)$ & $65(49.6)$ \\
& AA & $119(55.1)$ & $65(63.7)$ & $54(47.4)$ & $56(61.5)$ & $55(42.0)$ \\
\hline
\end{tabular}

$\mathrm{SNP}=$ single nucleotide polymorphism; $\mathrm{CHB}=$ chronic hepatitis $\mathrm{B} ; \mathrm{HBV}=$ Hepatitis B Virus; HCC = Hepatocellular carcinoma

${ }^{\text {a }}$ Compared with TC + CC genotypes (CHB with HCC versus CHB without HCC); OR $=25.00, P=0.0000048$.

b Compared with GA + GG genotypes (CHB with HCC versus CHB without HCC and healthy control); OR = 1.95, $P=0.016$ and $\mathrm{OR}=2.43, P=0.0009$, respectively. 
Table 4 Haplotype analysis for eight polymorphisms of autophagy genes.

\begin{tabular}{lllll}
\hline Haplotype & Freq. case & Freq. ctrl & $\chi^{2}$-value & $p$-value \\
\hline TACCTCGC & 0.1744 & 0.1955 & 0.3108 & 0.5772 \\
TATCTCGC & 0.07418 & 0.0986 & 0.8012 & 0.3707 \\
GACCTCGT & 0.02879 & 0.01787 & 0.5377 & 0.4634 \\
TACCTTGT & 0.08284 & 0.08328 & 0.000261 & 0.9871 \\
TACCTCGT & 0.02875 & 0.01648 & 0.6969 & 0.4038 \\
TACCTTGC & 0.1351 & 0.08487 & 2.656 & 0.1032 \\
TACTTCGC & 0.02811 & 0.01362 & 1.045 & 0.3066 \\
GACCTTGC & 0.04419 & 0.02855 & 0.7182 & 0.3968 \\
GACCTCGC & 0.03483 & 0.03202 & 0.02542 & 0.8733 \\
GATCTTGC & 0.0307 & 0.03039 & 0.00034 & 0.9853 \\
TGCCTTGC & 0.02956 & 0.04559 & 0.7585 & 0.3838 \\
GGCCTTGC & 0.0244 & 0.0174 & 0.2474 & 0.6189 \\
TATCTTGC & 0.03779 & 0.05445 & 0.6726 & 0.4122 \\
TGTCTTGC & 0.003865 & 0.02037 & 2.544 & 0.1107 \\
TGCCTCGC & 0.06116 & 0.06849 & 0.09337 & 0.7599 \\
GATCTCGC & 0.02769 & 0.03251 & 0.08423 & 0.7717 \\
TGCCTTGT & 0.01815 & 0.03068 & 0.7083 & 0.4000 \\
TATCTTGT & 0.04792 & 0.02907 & 0.9845 & 0.3211 \\
GGCCTCGC & 0.02489 & 0.04177 & 0.949 & 0.3300 \\
TGCTTTGC & 0.008688 & 0.0186 & 0.7912 & 0.3737 \\
TGTCTCGT & 0.009606 & 0.02651 & 1.765 & 0.1840 \\
TACCTCAC & 0.02364 & 0.008007 & 1.575 & 0.2095 \\
GGCCTCGT & 0.02068 & 0.005336 & 1.825 & 0.1767 \\
\hline
\end{tabular}

Table 5 Analysis of the interaction of ATG5 gene polymorphisms (rs41292420, rs77859116, and rs510432) using multifactor dimensionality reduction (MDR) approach.

\begin{tabular}{lcccl}
\hline Combination & w HCC & w/o HCC & C/C ratio & Pred. class \\
\hline CC, TT, CC & 16.0 & 21.0 & 0.7619 & Low risk \\
CC, TT, CT & 25.0 & 46.0 & 0.5435 & Low risk \\
CC, TT, TT & 13.0 & 15.0 & 0.8667 & Low risk \\
CC, CT, CC & 0.0 & 5.0 & 0.0 & Low risk \\
CC, CT, CT & 0.0 & 2.0 & 0.0 & Low risk \\
CC, CT, TT & 0.0 & 2.0 & 0.0 & Low risk \\
CT, TT, CC & 6.0 & 1.0 & 6.0 & High risk \\
CT, TT, CT & 5.0 & 5.0 & 1.0 & Low risk \\
CT, TT, TT & 0.0 & 5.0 & 0.0 & Low risk \\
CT, CT, CC & 0.0 & 1.0 & 0.0 & Low risk \\
TT, TT, CT & 0.0 & 1.0 & 0.0 & Low risk \\
\hline
\end{tabular}

${ }^{\mathrm{a}} \mathrm{C} / \mathrm{C}=$ Case/Control; ${ }^{\mathrm{b}}$ Pred. $=$ Predicted.

\section{DISCUSSION}

In this study, we determined the association between eight functional SNPs and the susceptibility to chronic HBV infection. These SNPs were located on the promoter region (mTOR rs2295080, ATG5 rs510432, IGF1 rs75960260, and PIK3C3 rs3813065), coding exon (ATG5 rs77859116 and ATG16L1 rs2241880), and exon-intron boundary
(ATG7 rs2305686 and ATG5 rs41292420). The results suggested that ATG5 rs77859116 and ATG16L1 rs2241880 polymorphisms might play an important role in the risk of HBV-related liver cancer development.

ATG5 is an important gene in the initiation of the autophagosome formation. It is activated by ATG7 and forms a complex with ATG12 and ATG16L $1^{12}$. Several studies have reported that ATG5 is associated with autoimmune diseases; such as, systemic lupus erythematosus. The $\mathrm{T}$ allele of ATG5 rs573775 has been reported to affect SLE susceptibility, cytokine production, and disease features $^{13,14}$. Furthermore, the ATG5 was associated with several kinds of cancer; such as, thyroid, prostate, stomach, and colon cancers ${ }^{15-18}$. This study showed that the T allele and TT genotype of ATG5 rs77859116 was associated with the risk to HCC. The rs77859116 polymorphism was mapped to chromosome $6 q 21$ on coding exon 3 and caused an amino acid exchange from isoleucine to valine. This non-synonymous SNP might affect the structure of the ATG5 protein to form a complex with ATG12 and ATG16L1, leading to an interruption of the autophagosome formation. This hypothesis is needed to be proven in a further study.

Although we did not find any association of the other two SNPs (rs41292420 and rs510432) of the ATG5 gene, it was revealed that there was significant association between the genotype combinations (CT (rs41292420), TT (rs77859116), and CC (rs510432) of the ATG5 and the risk to CHB with HCC. The ATG5 rs41292420 was mapped to the exon - intron boundary region. This position may affect either the exon skipping or utilization of the cryptic splice sites, resulting in the absence of normally spliced mRNA ${ }^{19}$. Another SNP, ATG5 rs510432 is on the first exon in the promoter region (C-335T). This variant has been reported to significantly enhance promoter activity ${ }^{20}$. The combination of these functional SNPs with others may have a risk effect to HBV-related liver disease.

The ATG16L1 gene encodes a protein that binds to the ATG12-ATG5 conjugate. It is a key regulatory autophagy gene involved in the elongation and closure steps of autophagosomes ${ }^{21}$. Several studies have reported that ATG16L1 polymorphism was associated with Crohn's disease ${ }^{22,23}$. Furthermore, the polymorphism of the ATG16L1 (rs2241880) has been shown to be associated with the susceptibility to gastric cancer, thyroid carcinoma, and palmoplantar pustulosis ${ }^{24-26}$. In this study, we found that the A allele and AA genotype of ATG16L1 SNP 
rs2241880 were associated with an increased risk in chronic HBV with HCC. This result suggested that ATG16L1 polymorphism might be the genetic factor responsible for the development of HCC.

The ATG16L1 rs2241880 was located on chromosome 2q37.1 on coding exon 9. This variant induced an amino acid exchange from the polar threonine to nonpolar alanine at the evolutionarily conserved position 300 of the $\mathrm{N}$ terminus in ATG16L1. The previous study showed macrophages derived from ATG16L1-deficient mice produced higher levels of IL-1 ${ }^{27}$. Although not yet proven, there were many studies showing the lack of IL- $1 \beta$ might increase the host susceptibility to viral infection and harmful inflammatory host responses ${ }^{28}$. These studies suggested that ATG16L1 might be related to the IL- $1 \beta$ transcription. Nevertheless, the functional consequences of rs2241880 remain unconfirmed at the time of conducting this study.

In conclusion, we found an association between the ATG5 and ATG16L1 polymorphisms and susceptibility to chronic HBV infection with the development of hepatocellular carcinoma. However, an association study using a larger sample size should be performed to verify the findings. This association on hepatocellular carcinoma was analysed only in Thai patients from only one hospital. It would be interesting to extend the analysis to different part of Thailand and different ethnic groups in the future. In addition, there is still no report on the functional study of the polymorphisms in these two genes. Thus functional analysis involving the effect of these polymorphisms is required in the future.

Acknowledgements: This study was supported in part by a Graduate Program Scholarship from the Graduate School, Kasetsart University, Kasetsart University Research and Development Institute (KURDI), and Faculty of Science, Kasetsart University. This study was also funded by the National Research University (NRU59-026-HR), Chulalongkorn University.

\section{REFERENCES}

1. Yue M, Mingshou H (2017) Current epidemiology, prevention and treatment of hepatitis B in China. Infect Dis Trop Med 3, e412.

2. Lin TM, Chen CJ, Wu MM, Yang CS, Chen JS, Lin CC, Kwang TY, Hsu ST, et al (1989) Hepatitis B virus markers in Chinese twins. Anticanc Res 9, 737-41.

3. Feng Z (2010) p53 Regulation of the IGF-1/AKT/ mTOR pathways and the endosomal compartment. Cold Spring Harb Perspect Biol 2, a001057.

4. Mai S, Muster B, Bereiter-Hahn J, Jendrach M (2012) Autophagy proteins LC3B, ATG5 and ATG12 partici- pate in quality control after mitochondrial damage and influence lifespan. Autophagy 8, 47-62.

5. Maiuri MC, Zalckvar E, Kimchi A, Kroemer G (2007) Self-eating and self-killing: crosstalk between autophagy and apoptosis. Nat Rev Mol Cell Biol 8, 741-52.

6. Tantithavorn V, Hirankarn N, Tangkijvanich P, Kimkong I (2015) Expression profile of ATG16L1 and mTOR genes in hepatitis B virus infection. In: Proceedings of the 53rd Kasetsart Univ Annual Conference, Bangkok.

7. Kunanopparat A, Kimkong I, Palaga T, Tangkijvanich P, Sirichindakul B, Hirankarn N (2016) Increased ATG5-ATG12 in hepatitis B virus-associated hepatocellular carcinoma and their role in apoptosis. World J Gastroenterol 22, 8361-74.

8. Kunanopparat A, Hirankarn N, Kittigu C, Tangkijvanich P, Kimkong I (2016) Autophagy machinery impaired interferon signaling pathways to benefit hepatitis B virus replication. Asian Pac J Allergy Immunol 34, 77-85.

9. Miller SA, Dykes DD, Polesky HF (1988) A simple salting out procedure for extracting DNA from human nucleated cells. Nucleic Acids Res 16, 1215.

10. Purcell S, Neale B, Todd-Brown K, Thomas L, Ferreira MAR, Bender D, Maller J, Sklar P, et al (2007) PLINK: a tool set for whole-genome association and population-based linkage analyses. Am J Hum Genet 81, 559-75.

11. Hahn LW, Ritchie MD, Moore JH (2003) Multifactor dimensionality reduction software for detecting gene-gene and gene-environment interactions. Bioinformatics 19, 376-82.

12. Kuma A, Hatano M, Matsui M, Yamamoto A, Nakaya $\mathrm{H}$, Yoshimori T, Ohsumi Y, Tokuhisa T, et al (2004) The role of autophagy during the early neonatal starvation period. Nature 432, 1032-6.

13. International Consortium for Systemic Lupus Erythematosus Genetics (SLEGEN), Harley JB, AlarcónRiquelme ME, Criswell LA, Jacob CO, Kimberly RP, Moser KL, Tsao BP, et al (2008) Genome-wide association scan in women with systemic lupus erythematosus identifies susceptibility variants in ITGAM, PXK, KIAA1542 and other loci. Nat Genet 40, 204-10.

14. López P, Alonso-Pérez E, Rodríguez-Carrio J, Suárez A (2013) Influence of Atg5 mutation in SLE depends on functional IL-10 genotype. PLoS One 8, e78756.

15. Plantinga TS, van de Vosse E, Huijbers A, Netea MG, Joosten LAB, Smit JWA, Netea-Maier RT (2014) Role of genetic variants of autophagy genes in susceptibility for non-medullary thyroid cancer and patients outcome. PLoS One 9, e94086.

16. Kim MS, Song SY, Lee JY, Yoo NJ, Lee SH (2011) Expressional and mutational analyses of ATG5 gene in prostate cancers. APMIS 119, 802-7.

17. An CH, Kim MS, Yoo NJ, Park SW, Lee SH (2011) Mutational and expressional analyses of ATG5, an 
autophagy-related gene, in gastrointestinal cancers. Pathol Res Pract 207, 433-7.

18. Kang MR, Kim MS, Oh JE, Kim YR, Song SY, Kim SS, Ahn CH, Yoo NJ, et al (2009) Frameshift mutations of autophagy-related genes ATG2B, ATG5, ATG9B and ATG12 in gastric and colorectal cancers with microsatellite instability. $J$ Pathol 217, 702-6.

19. Carlton VEH, Ireland JS, Useche F, Faham M (2006) Functional single nucleotide polymorphism-based association studies. Hum Genom 2, 391-402.

20. Martin LJ, Gupta J, Jyothula SS, Butsch Kovacic M, Biagini Myers JM, Patterson TL, Ericksen MB, He H, et al (2012) Functional variant in the autophagyrelated 5 gene promotor is associated with childhood asthma. PLoS One 7, e33454.

21. Levine B, Mizushima N, Virgin HW (2011) Autophagy in immunity and inflammation. Nature 469, 323-35.

22. Rioux JD, Xavier RJ, Taylor $\mathrm{KD}$, Silverberg MS, Goyette P, Huett A, Green T, Kuballa P, et al (2007) Genome-wide association study identifies new susceptibility loci for Crohn disease and implicates autophagy in disease pathogenesis. Nat Genet 39, 596-604.

23. Koder S, Repnik K, Ferkolj I, Pernat C, Skok P, Weersma RK, Potočnik U (2015) Genetic polymorphism in ATG16L1 gene influences the response to adalimumab in Crohn's disease patients. Pharmacogenomics 16, 191-204.

24. Burada F, Ciurea ME, Nicoli R, Streata I, Vilcea ID, Rogoveanu I, Ioana M (2016) ATG16L1 T300A polymorphism is correlated with gastric cancer susceptibility. Pathol Oncol Res 22, 317-22.

25. Huijbers A, Plantinga TS, Joosten LA, Aben KK, Gudmundsson J, den Heijer M, Kiemeney LA, Netea MG, et al (2012) The effect of the ATG16L1 Thr300Ala polymorphism on susceptibility and outcome of patients with epithelial cell-derived thyroid carcinoma. Endocr Relat Canc 19, L15-8.

26. Douroudis K, Kingo K, Traks T, Rätsep R, Silm H, Vasar E, Kõks S (2011) ATG16L1 gene polymorphisms are associated with palmoplantar pustulosis. Hum Immunol 72, 613-5.

27. Saitoh T, Fujita N, Jang MH, Uematsu S, Yang BG, Satoh T, Omori H, Noda T, et al (2008) Loss of the autophagy protein Atg16L1 enhances endotoxininduced IL-1 $\beta$ production. Nature 456, 264-8.

28. Shoji-Kawata S, Levine B (2009) Autophagy, antiviral immunity, and viral countermeasures. Biochim Biophys Acta 1793, 1478-84. 